\title{
Generalized Spin Structures on Four Dimensional Space-Times
}

\author{
S. J. Avis and C. J. Isham \\ Theoretical Physics Group, Blackett Laboratory, Imperial College of Science and Technology, \\ London, England
}

\begin{abstract}
We discuss various methods for investigating the existence and uniqueness of generalized spin structures. We show that on a four dimensional manifold whole families may be constructed using any internal symmetry group of the form $G / \mathbb{Z}_{2}$, where $G$ is a simply connected Lie group.
\end{abstract}

\section{Introduction}

Much of the current work in quantum gravity is concerned with the construction of classical and quantized fields propagating on a fixed background space-time $M$. This subject is of interest in its own right and also occurs in the evaluation of the one loop contribution to a functional integral approach to quantum gravity proper. An especially intriguing aspect is the rôle played by the global topology of the space-time.

In the present paper we will concentrate on aspects of space-time topology that are reflected in spinor field theory. There will in general be a number of inequivalent spinor structures [13] and these are classified by elements of the cohomology group $H^{1}\left(M ; \mathbb{Z}_{2}\right)$. We have previously discussed a number of features of this phenomenon relevant to quantum field theory [12], [2]. However the very existence of spinors is determined by global topological properties of $M$; specifically a necessary and sufficient condition is the vanishing of the second Stiefel Whitney class of the tangent bundle [13]. The problem of handling spacetimes which do not satisfy this restriction has been approached in various ways. We wish to focus on the ideas of Whiston [18], Hawking et al. [8], Back et al. [4], and Forger et al. [7], and discuss and extend their techniques. Hawking and Pope sought to replace the $\operatorname{Spin}(4)$ group covering $\mathrm{SO}(4)$ with $\operatorname{Spin}^{c}(4)=$ Spin (4) $\times_{\mathbb{Z}_{2}} U(1)$ whilst Back et al. employed Spin (4) $\times_{\mathbb{Z}_{2}} G$ where $G$ was basically $\mathrm{SU}(2)$. The $\operatorname{Spin}^{c}(4)$ method is limited to a special class of space-times whereas the construction of Back et al, although free of this defect, represents only one of a large class of Spin (4) $\times_{\mathbb{Z}_{2}} G$ covering techniques. We will develop some of these in Sec. 4 and to motivate and clarify the method employed we will first discuss the existing work in Sect. 2 and 3 using the appropriate mathematical language. 
These new structures exist on any four dimensional manifold and can employ any internal gauge group of the form $G / \mathbb{Z}_{2}$ where $G$ is simply connected. We will show that inequivalent generalized spin structures of this type are related to the groups $H^{4}\left(M ; \pi_{3}(G)\right)$ and $H^{1}\left(M ; \mathbb{Z}_{2}\right)$. We have concentrated on $\mathrm{SO}(4)$ rather than $\mathrm{SO}_{\uparrow}(3,1)$ (i.e., we have in mind the riemannian instanton contributions to a functional integral) because the problem is more severe here. However we will add paranthetical remarks on the $\operatorname{SO}_{\uparrow}(3,1)$ case in appropriate places. Our main interest is correspondingly when $M$ is a compact oriented manifold.

\section{Spin Structures}

2.1. We will start by reviewing the standard material on the existence and uniqueness of spin structures [13]. The Lie group $\mathrm{SO}(n)(n \geqq 3)$ possesses a simply connected double covering group $\operatorname{Spin}(n)$ with a group homomorphism $\Lambda: \operatorname{Spin}(n) \longrightarrow$ $\mathrm{SO}(n)$. Let $\xi$ be a principal $\mathrm{SO}(n)$ bundle over a manifold $M$. Then a $\operatorname{Spin}(n)$ structure consists of a pair $(\eta, f)$ where $\eta$ is a principal $\operatorname{Spin}(n)$ bundle over $M$ and $f$ is a bundle map from the bundle space $E(\eta)$ onto $E(\xi)[11]$

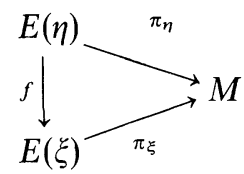

The group actions on the two spaces are required to be compatible:

$$
f(p A)=f(p)[A] \quad \forall p \in E(\eta), \quad \forall A \in \operatorname{Spin}(n)
$$

where $[A]=\Lambda(A)$

An equivalent formulation is the following. The double coverings (i.e. principal $\mathbb{Z}_{2}$-bundles) over a topological space $X$ are classified by the elements of the cohomology group $H^{1}\left(X ; \mathbb{Z}_{2}\right)=\operatorname{Hom}\left(\pi_{1}(X), \mathbb{Z}_{2}\right)[16]$. The $\mathbb{Z}_{2}$ coverings of a Lie group are themselves Lie groups and in particular, since $H^{1}\left(\mathrm{SO}(n) ; \mathbb{Z}_{2}\right)=\mathbb{Z}_{2}$, there are just two double coverings of $\mathrm{SO}(n)$; the trivial one, $\mathrm{SO}(n) \times \mathbb{Z}_{2}$, and the non trivial $\operatorname{Spin}(n)$ covering, represented respectively by the identity element $e$ and the generator $\alpha$ of $H^{1}\left(\mathrm{SO}(n) ; \mathbb{Z}_{2}\right)$ A spin structure over $M$ is equivalent to a double covering $\sigma$ of $E(\xi)$ which, when restricted to every $\mathrm{SO}(n)$ fibre, reproduces the non trivial Spin $(n)$ covering of $\mathrm{SO}(n)$; because $M$ is path connected it is sufficient to demand this on only one fibre, it is then automatically true for the others. Since $\sigma$ is represented by an element of $H^{1}\left(E(\xi) ; \mathbb{Z}_{2}\right)$, this condition is simply

$$
i^{*}(\sigma)=\alpha
$$

where $i$ denotes the injection of the standard $\mathrm{SO}(n)$ fibre over the basepoint in $M$. The various $\mathbb{Z}_{2}$ cohomology groups of $\xi$ fit together in the Serre short exact sequence;

$$
0 \longrightarrow H^{1}\left(M ; \mathbb{Z}_{2}\right) \stackrel{\pi_{\xi}^{*}}{\longrightarrow} H^{1}\left(E(\xi) ; \mathbb{Z}_{2}\right) \stackrel{i^{*}}{\longrightarrow} H^{1}\left(\mathrm{SO}(n) ; \mathbb{Z}_{2}\right) \stackrel{{ }^{\tau}}{\longrightarrow} H^{2}\left(M ; \mathbb{Z}_{2}\right)
$$

By exactness of (2.4), the condition (2.3) is satisfied if and only if $\tau(\alpha)=0$. How- 
ever $\tau(\alpha)$ is equal to $w_{2}(\xi)$ - the second Stiefel Whitney class of $\xi$ - and hence the well known necessary and sufficient condition for a $\operatorname{Spin}(n)$ structure to exist is

$$
\omega_{2}(\xi)=0
$$

If $\sigma_{1}, \sigma_{2} \in H^{1}\left(E(\xi) ; \mathbb{Z}_{2}\right)$ both represent spin structures then $i^{*}\left(\sigma_{1}-\sigma_{2}\right)=0$ and so, by the exactness of (2.4), $\sigma_{1}-\sigma_{2}=\pi_{\xi}^{*}(\beta)$ for some $\beta$ in $H^{1}\left(M ; \mathbb{Z}_{2}\right)$. Thus the different possible spin structures are labelled (after choosing some fixed $\sigma_{1}$ ) by the elements of $H^{1}\left(M ; \mathbb{Z}_{2}\right)$. A priori, these inequivalent structures could appear as either inequivalent bundles $\eta$ or maps $f$ in (2.1). These two features are reflected in different ways when the spinor fields, defined as cross sections of associated vector bundles, are quantized. The modes of the Dirac equation depend on the choice of bundle as do the corresponding Green's functions which appear in a perturbative approach to the quantum field theory. At a rigorous level the test functions employed to smear the operator fields must be cross sections of the dual vector bundle. On the other hand the various choices of bundle maps $f$ in (2.1) lead to different spin connections (which affects the Dirac equation again) since these are defined in $E(\eta)$ as the pull back of the fixed connection in $E(\xi)$.

2.2. Some insight into the classification of different spin bundles can be obtained by studying the fibration

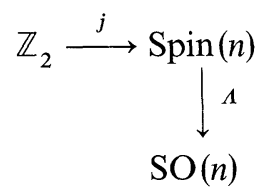

which, since $\mathbb{Z}_{2}$ is an invariant subgroup of $\operatorname{Spin}(n)$, leads to the derived fibration [5]

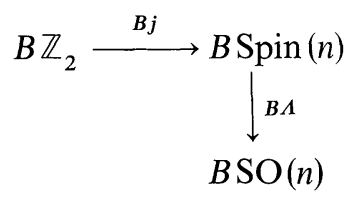

where $B G$ denotes the base space of a universal $G$ bundle $[16]$. Now $B \mathbb{Z}_{2}$ is an Eilenberg Maclane space $[15] K\left(\mathbb{Z}_{2}, 1\right)$ with the characteristic property

$$
\begin{aligned}
& \pi_{1}\left(K\left(\mathbb{Z}_{2}, 1\right)\right)=\mathbb{Z}_{2} \\
& \pi_{i}\left(K\left(\mathbb{Z}_{2}, 1\right)\right)=0
\end{aligned} \quad i \geqq 2
$$

Furthermore if $\pi$ is any abelian group

$$
[M, K(\pi, n)]=H^{n}(M ; \pi)
$$

and $\quad[M, B G]=\mathscr{B}_{G}(M)$

where $[M, X]$ denotes the homotopy classes of (pointed) maps from $M$ into $X$ and $\mathscr{B}_{G}(M)$ is the set of isomorphism classes of principal $G$ bundles over $M$. The fibration in (2.7) may be obtained as the pull back of a certain universal bundle 
over $K\left(\mathbb{Z}_{2}, 2\right)$ leading to the diagram

$$
\begin{aligned}
& K\left(\mathbb{Z}_{2}, 1\right) \stackrel{\boldsymbol{B j}^{\prime}}{\longrightarrow} B \operatorname{Spin}(n) \\
& \downarrow^{\boldsymbol{B} \Lambda} \\
& B \mathrm{SO}(n) \stackrel{\theta}{\longrightarrow} K\left(\mathbb{Z}_{2}, 2\right)
\end{aligned}
$$

which as explained in $[3,14]$, gives rise to the exact sequence of pointed sets

$$
\begin{aligned}
& \cdots \longrightarrow[M, \operatorname{Spin}(n)] \stackrel{\Lambda_{*}}{\longrightarrow}[M, \operatorname{SO}(n)] \stackrel{\Omega \theta_{*}}{\longrightarrow} H^{1}\left(M ; \mathbb{Z}_{2}\right) \stackrel{B j *}{\longrightarrow} \mathscr{B}_{\operatorname{Spin}(n)}(M) \\
& \stackrel{B \Lambda *}{\longrightarrow} \mathscr{B}_{\mathrm{SO}(n)}(M) \stackrel{\theta_{*}}{\longrightarrow} H^{2}\left(M ; \mathbb{Z}_{2}\right)
\end{aligned}
$$

An SO $(n)$ bundle $\xi$ may be covered by a Spin $(n)$ bundle if and only if it lies in the image of $B \Lambda_{*}$ or equivalently (by exactness) if $\theta_{*}(\xi)=0$. Thus the cohomology element $\theta_{*}(\xi)$ measures the obstruction to constructing a Spin $(n)$ structure. Since $\theta_{*}(\xi)=w_{2}(\xi)$ we recover the condition $(2.5)$. Note that (2.12) also provides information on the homotopy classes of $\mathrm{SO}(n)$ gauge functions with the equivalence relation

$$
\Omega_{1} \equiv \Omega_{2} \quad \text { iff } \quad \Omega_{1} \Omega_{2}^{-1} \quad \text { can be lifted to } \operatorname{Spin}(n)
$$

These may be classified by

$$
\begin{aligned}
T_{\xi} & =[M, \mathrm{SO}(n)] / m \Lambda_{*}=[M, \mathrm{SO}(n)] / \operatorname{ker} \Omega \theta_{*} \\
& \approx m \Omega \theta_{*}=\operatorname{ker} B j_{*}
\end{aligned}
$$

If we focus on the tangent bundle the analogue in a lorentzian space-time of $\operatorname{Spin}(4)$ is $\operatorname{SL}(2, \mathbb{C})$. However $\mathscr{B}_{S L(2, \mathbb{C})}(M)=H^{4}(M ; \mathbb{Z})[3]$ which vanishes if $M$ is noncompact and hence in this situation all $\operatorname{SL}(2, \mathbb{C})$ bundles are necessarily trivial and $\operatorname{Im} B j_{*}=0$ in (2.12). Thus all the topological information concerning inequivalent spinor structures is carried by the bundle maps $f$ and hence by the different spin connections.

On a compact manifold we can employ the Serre cohomology sequence of (2.11) to show that

$$
B \Lambda^{*}: H^{4}(B \operatorname{SO}(n) ; \mathbb{R}) \longrightarrow H^{4}(B \operatorname{Spin}(n) ; \mathbb{R})
$$

is an isomorphism. However on a four manifold [3]

$$
\mathscr{B}_{\operatorname{Spin}(n)}(M)= \begin{cases}H^{4}(M ; \mathbb{Z}) & n \neq 4 \\ H^{4}(M ; \mathbb{Z}) \oplus H^{4}(M ; \mathbb{Z}) & n=4\end{cases}
$$

and so in fact a $\operatorname{Spin}(n)$ bundle $\eta$ is uniquely determined by its real second Chern class (there are two of them if $n=4$ ) which according to (2.14) is itself uniquely determined by the Pontryagin class (plus the Euler class if $n=4$ ) of the $\operatorname{SO}(n)$ bundle which it covers. In particular $\eta$ is uniquely determined by $\xi$ and once again $B j_{*}=0$ in (2.12).

Thus on four dimensional manifolds the spin bundles are all isomorphic and different spin structures are obtained by running through the classes of inequivalent covering maps, $f$. These correspond to choosing inequivalent spinor connections 
and hence Dirac Lagrangians: the quantum field theory implications of having inequivalent spin structures are discussed in reference [2].

\section{3. $\operatorname{Spin}^{c}$ Structures}

3.1. If $w_{2}(\xi) \neq 0$ then one might attempt to cover $\xi$ with a bundle whose structure group was not $\operatorname{Spin}(n)$ but which was related to it in some useful and interesting way. It has been proposed in [18] and [8] to employ the group $\operatorname{Spin}^{c}(4)$ in the particular case that $\xi$ is the tangent bundle. The group $\operatorname{Spin}^{c}(4)$ is defined as [1]

$$
\operatorname{Spin}^{c}(4)=\operatorname{Spin}(4) \times{ }_{\mathbb{Z}_{2}} U(1)
$$

with the $\mathbb{Z}_{2}$ equivalence relation

$$
(x, u) \equiv(-x,-u) \quad x \in \operatorname{Spin}(4), u \in U(1)
$$

in which the minus signs may be conveniently interpreted by viewing Spin(4)= $\mathrm{SU}(2) \times \mathrm{SU}(2)$ as a matrix group and $U(1)$ as the multiplicative group of unit modulus complex numbers. $U(1)$ is an invariant, closed, subgroup of $\operatorname{Spin}^{c}(4)$ :

$$
\begin{gathered}
U(1) \stackrel{c}{\longrightarrow} \operatorname{Spin}^{c}(4) \\
u \longrightarrow[1, u]
\end{gathered}
$$

and $\operatorname{Spin}^{c}(4) / U(1) \approx \mathrm{SO}(4)$ with the projection

$$
\begin{aligned}
\operatorname{Spin}^{c}(4) & \longrightarrow \mathrm{SO}(4) \\
{[x, u] } & \longmapsto[x]=\Lambda(x)
\end{aligned}
$$

leading to the fibre bundle

$$
U(1) \longrightarrow \operatorname{Spin}^{c}(4) \longrightarrow \mathrm{SO}(4)
$$

Proceeding by analogy with the Spin(4) case one observes that the $U(1)$ bundles over any manifold $X$ are classified by the elements of the cohomology group $H^{2}(X ; \mathbb{Z})[9]$. In particular, on recalling that $\mathrm{SO}(4) \approx \$^{3} \times \mathbb{R} P^{3}$, we may readily compute

$$
H^{2}(\mathrm{SO}(4) ; \mathbb{Z})=\mathbb{Z}_{2}=\{e, \alpha\}
$$

and so there are two isomorphism classes of principal $U(1)$ bundles over $\mathrm{SO}(4)$ with the trivial class including $\mathrm{SO}(4) \times U(1)$. Now the exact homotopy sequence of any $U(1)$ bundle over $\mathrm{SO}(4)$

$$
U(1) \longrightarrow E \longrightarrow \mathrm{SO}(4)
$$

shows that $\pi_{1}(E)=\mathbb{Z}$ or $\mathbb{Z} \oplus \mathbb{Z}_{2}$. However there is a homeomorphism

$$
\begin{aligned}
\chi: \operatorname{Spin}^{c}(4) & \longrightarrow U(1) / \mathbb{Z}_{2} \\
{[x, u] } & \longmapsto[u]
\end{aligned}
$$

and a fibration

$$
\operatorname{Spin}(4) \stackrel{\varphi}{\longrightarrow} \operatorname{Spin}^{c}(4) \stackrel{x}{\longrightarrow} U(1) / \mathbb{Z}_{2}
$$


with

$$
\begin{aligned}
\varphi: \operatorname{Spin}(4) & \longrightarrow \operatorname{Spin}^{c}(4) \\
x & \longmapsto[x, 1]
\end{aligned}
$$

Since $U(1) / \mathbb{Z}_{2} \approx \mathbb{S}^{1}$ and since $\operatorname{Spin}(4)$ bundles over $\mathbb{S}^{n}$ are classified by $\Pi^{n-1}$ (Spin(4)) [16] it follows that (3.9) is trivial and hence topologically-but not as Lie groups $-\operatorname{Spin}^{c}(4) \approx \operatorname{Spin}(4) \times U(1)$. In particular $\pi_{1}\left(\operatorname{Spin}^{c}(4)\right)=\mathbb{Z}$ and so the fibration of (3.5) must correspond to the non trivial generator $\alpha$ of $H^{2}(\mathrm{SO}(4)$; $\mathbb{Z})=\mathbb{Z}_{2}$. (The trivial $U(1)$ bundle has $\left.\pi_{1}(\operatorname{SO}(4) \times U(1))=\mathbb{Z} \oplus \mathbb{Z}_{2}\right)$.

It is therefore natural to define a $\operatorname{Spin}^{c}(4)$ structure on $M$ as a $U(1)$ bundle over $E(\xi)$ which, when restricted to every $\mathrm{SO}(4)$ fibre, reproduces a $U(1)$ bundle belonging to the nontrivial class represented by $\alpha$. (Since $U(1)$ is in the centre of $\operatorname{Spin}^{c}(4)$ every such bundle can be given a unique $\operatorname{Spin}^{c}(4)$ group structure). The relevant short exact sequence is (noting $\left.H^{1}(\mathrm{SO}(4) ; \mathbb{Z})=\{0\}\right)$;

$$
0 \longrightarrow H^{2}(M ; \mathbb{Z}) \stackrel{\pi^{*}}{\longrightarrow} H^{2}(E(\xi) ; \mathbb{Z}) \stackrel{i^{*}}{\longrightarrow} H^{2}(\operatorname{SO}(4) ; \mathbb{Z}) \stackrel{\tau}{\longrightarrow} H^{3}(M ; \mathbb{Z})
$$

Clearly $\sigma \in H^{2}(E(\xi) ; \mathbb{Z})$ represents a $\operatorname{Spin}^{c}(4)$ structure if and only if $i^{*}(\sigma)=\alpha$ or equivalently $\tau(\alpha)=0$. However $\tau(\alpha)=W_{3}(\xi)$ - the third integral Stiefel Whitney class of $\xi$-and hence one obtains the standard [9] and [10] necessary and sufficient condition for the existence of a Spinc(4) structure

$$
W_{3}(\xi)=0
$$

This shows that it is not always possible to employ the $\operatorname{Spin}^{c}(4)$ group. Of course if $M$ is simply connected and compact then $H^{3}(M ; \mathbb{Z})=H_{1}(M ; \mathbb{Z})=\{0\}$ and (3.12) is automatically satisfied.

3.2. It is clear from (3.11) that the difference between two $\operatorname{Spin}^{c}(4)$ structures can be represented by an element of $H^{2}(M ; \mathbb{Z})$ and, as in the Spin $(n)$ case, it is instructive to enquire when different $\operatorname{Spin}^{c}(4)$ structures are associated with different bundles. The fibre bundle (3.5) leads to the fibration (cf. (2.7))

$$
B U(1) \stackrel{B k}{\longrightarrow} B \operatorname{Spin}^{c}(4) \stackrel{B \rho}{\longrightarrow} B \mathrm{SO}(4)
$$

which, since $B U(1)=K(\mathbb{Z}, 2)$ gives the Puppe sequence (cf. 2.12)

$$
\begin{gathered}
\cdots \longrightarrow\left[M, \operatorname{Spin}^{c}(4)\right] \stackrel{\rho_{*}}{\longrightarrow}[M, \mathrm{SO}(4)] \stackrel{\Omega \theta_{*}}{\longrightarrow} H^{2}(M ; \mathbb{Z}) \stackrel{B k_{*}}{\longrightarrow} \mathscr{B}_{\operatorname{Spin}^{c}(4)}(M) \\
\stackrel{B \rho_{*}}{\longrightarrow} \mathscr{B}_{\mathrm{SO}(4)}(M) \stackrel{\theta_{*}}{\longrightarrow} H^{3}(M ; \mathbb{Z})
\end{gathered}
$$

where as might be anticipated $\theta_{*}(\xi)=W_{3}(\xi)$, thus recovering (3.12).

The Postnikov factorization method used in [3] may be easily applied (see Sect. 4 for a short discussion) to show that

$$
\mathscr{B}_{\operatorname{Spin}^{c}(4)}(M)=H^{2}(M ; \mathbb{Z}) \oplus H^{4}(M ; \mathbb{Z}) \oplus H^{4}(M ; \mathbb{Z})
$$


where one uses

$$
\begin{aligned}
& \pi_{1}\left(B \operatorname{Spin}^{c}(4)\right)=0 \\
& \pi_{2}\left(B \operatorname{Spin}^{c}(4)\right)=\pi_{1}\left(\operatorname{Spin}^{c}(4)\right)=\mathbb{Z} \\
& \pi_{3}\left(B \operatorname{Spin}^{c}(4)\right)=\pi_{2}\left(\operatorname{Spin}^{c}(4)\right)=0 \\
& \left.\pi_{4}\left(B \operatorname{Spin}^{c}(4)\right)=\pi_{3}(4)\right)=\mathbb{Z} \oplus \mathbb{Z}
\end{aligned}
$$

The characteristic element in $H^{2}(M ; \mathbb{Z})$ is the first Chern class of the $U(1)$ bundle associated with the $\operatorname{Spin}^{c}(4)$ bundle via the homomorphism (cf.3.8)

$$
\begin{aligned}
l: \operatorname{Spin}^{c}(4) & \longrightarrow U(1) \\
{[x, u] } & \longrightarrow u^{2}
\end{aligned}
$$

(of course $U(1) / \mathbb{Z}_{2}$ is isomorphic to $U(1)$ via the map $u \longrightarrow u^{2}$ ). Now the subgroup embedding $k$ in (3.3) associates a $\operatorname{Spin}^{c}(4)$ bundle with any given $U(1)$ bundle and the composite of $k$ with $l$ is

$$
\begin{aligned}
l \cdot k: U(1) & \longrightarrow U(1) \\
u & \longmapsto u^{2}
\end{aligned}
$$

Consequently the map $B k_{*}$ in (3.14) is simply

$$
\begin{aligned}
H^{2}(M ; \mathbb{Z}) & \longrightarrow H^{2}(M ; \mathbb{Z}) \oplus H^{4}(M ; \mathbb{Z}) \oplus H^{4}(M ; \mathbb{Z}) \\
c & \longrightarrow(2 c, 0,0)
\end{aligned}
$$

Since (3.14) is only exact in the sense of pointed sets, the strict result of (3.14) and (3.19) is that the inequivalent $\operatorname{Spin}^{c}$ (4) bundles covering the trivial SO(4) bundle $\xi_{T}$ are labelled by

$$
D_{\xi_{T}}=\operatorname{ker} B \rho_{*}=\operatorname{im} B k_{*}=2 H^{2}(M ; \mathbb{Z})=H^{2}(M ; \mathbb{Z}) / \beta\left(H^{1}\left(M ; \mathbb{Z}_{2}\right)\right)
$$

where $\beta$ is the Bockstein map

$$
\begin{aligned}
\ldots \longrightarrow & H^{1}\left(M ; \mathbb{Z}_{2}\right) \stackrel{\beta}{\longrightarrow} H^{2}(M ; \mathbb{Z}) \stackrel{x^{2}}{\longrightarrow} H^{2}(M ; \mathbb{Z}) \stackrel{\bmod 2}{\longrightarrow} \\
& H^{2}\left(M ; \mathbb{Z}_{2}\right) \stackrel{\beta}{\longrightarrow} H^{3}(M ; \mathbb{Z})
\end{aligned}
$$

In particular if $H^{2}(M ; \mathbb{Z})$ has no two-torsion then

$$
D_{\xi}=H^{2}(M ; \mathbb{Z})
$$

In general one can show that the characteristic $H^{4}(M ; \mathbb{Z}) \oplus H^{4}(M ; \mathbb{Z})$ elements for any bundle $\eta$ covering an arbitrary $\xi$ are uniquely fixed by $\xi$ and hence the arbitrariness in the choice of $\eta$ is determined by the characteristic class $C$ in the $H^{2}(M ; \mathbb{Z})$ part of $\mathscr{B}_{\operatorname{Spin}^{c}(4)}(M)$. This may be chosen freely subject only to the condition

$$
C \bmod 2=\omega_{2}(\xi)
$$

Thus the classes $C_{1}, C_{2}$ for two different bundles must obey $\left(C_{1}-C_{2}\right) \bmod 2=0$ or (by 3.21)) $C_{1}=C_{2}+2 C$ for some $C$ in $H^{2}(M ; \mathbb{Z}$ ). This essentially reproduces the classification in (3.20) for any $\mathrm{SO}(4)$ bundle $\xi$. Note that by the exactness of (3.21) the existence of $C$ satisfying (3.23) is equivalent to $\beta\left(w_{2}(\xi)\right)=0$ which is just (3.12) again since $W_{3}(\xi)=\beta\left(w_{2}(\xi)\right)$. 
In summary all of the topological information concerning inequivalent $\operatorname{Spin}^{c}$ (4) structures (apart from 2-torsion) is coded in the different bundles: contrary to the situation pertaining in the Spin(4) case. Any bundle in $D_{\xi}$ is classified by the first Chern class which, using real coefficients, may be represented by the curvature two form of any connection in the corresponding $U(1)$ bundle. In a quantum field theory context these different bundles will be manifested as different ' $U(1)$ instanton' sectors and the two forms will for example appear in the right hand side of the axial current anomaly. This has been explicitly shown in [8] for $M=\mathbb{C P} 2$ although, since $H^{2}(\mathbb{C} P 2 ; \mathbb{Z})=\mathbb{Z}$, the $\operatorname{Spin}^{c}(4)$ structure in [8] represents only one of a countable family of possible ones.

The 2-torsion contribution is related to $\mathrm{SO}(4)$ gauge functions that are not liftable to $\operatorname{Spin}^{c}(4)$. The classes of these are defined (cf. (2.13))

$$
\begin{aligned}
T_{\xi} & =[M, \mathrm{SO}(4)] / m \rho_{*}=[M, \mathrm{SO}(4)] / \operatorname{ker} \Omega W_{3} \\
& =m \Omega W_{3}=\operatorname{ker} B k_{*}=\beta\left(H^{1}\left(M ; \mathbb{Z}_{2}\right)\right)
\end{aligned}
$$

\section{Generalized Spin Structures}

4.1. In order to avoid the topological restriction $W_{3}(\xi)=0$ it is natural to contemplate using a group of the form $\operatorname{Spin}_{G}(4)=\operatorname{Spin}(4) \times{ }_{\mathbb{Z}_{2}} G$ where $G$ is any Lie group with a $\mathbb{Z}_{2}=\{e, a\}$ in its centre. The $\mathbb{Z}_{2}$ equivalence relation on $\operatorname{Spin}(4) \times G$ is

$$
(x, g) \equiv(-x, a g)
$$

with the projection (cf. 3.4)

$$
\begin{aligned}
\rho: \operatorname{Spin}_{G}(4) & \longrightarrow \mathrm{SO}(4) \\
{[x, g] } & \longmapsto \Lambda(x)
\end{aligned}
$$

A $\operatorname{Spin}_{G}(4)$ structure may be defined as a $\operatorname{Spin}_{G}(4)$ bundle $\eta$ plus a bundle map $f: E(\eta) \longrightarrow E(\xi)$ such that

$$
f(p A)=f(p) \rho(A) \quad \forall p \in E(\eta), \forall A \in \operatorname{Spin}_{G}(4)
$$

Two such structures $(\eta, f)$ and $\left(\eta^{\prime}, f^{\prime}\right)$ are defined to be equivalent if there exists a $\operatorname{Spin}_{G}(4)$ bundle map $g: \eta \rightarrow \eta^{\prime}$ making the diagram

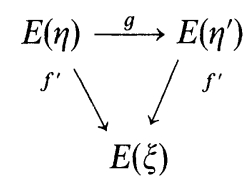

commute. (A similar equivalence relation is to be understood in the $\operatorname{Spin}(n)$ and $\operatorname{Spin}^{c}(n)$ cases).

Back et al. [4] found an example by choosing $G=\mathrm{SU}(2)$ and considering the projection $v$ of $\operatorname{Spin}(4)=\mathrm{SU}(2) \times \mathrm{SU}(2)$ onto $G$ viewed as one of the two $\mathrm{SU}(2)$ factors. This leads to a homomorphism

$$
\begin{aligned}
\mu: \mathrm{SO}(4) & \longrightarrow \operatorname{Spin}_{G}(4) \\
\Lambda(x) & \longmapsto[x, v(x)]
\end{aligned}
$$


and enables a $\operatorname{Spin}_{G}(4)$ bundle $\eta$ to be constructed which is associated with $\xi$. Since $\rho$ is a left inverse of $\mu$ this does indeed define a $\operatorname{Spin}_{G}(4)$ structure for an arbitrary four-manifold and the method has recently been extended [7] to encompass groups other than $\mathrm{SU}(2)$.

However, this technique only produces a small sample of the set of $\operatorname{Spin}_{G}(4)$ structures. The construction also differs in one significant respect from that of a Spin $^{c}(4)$ structure. Because of (4.4) the $\mathrm{SO}(4)$ bundle $\xi$ is actually a sub-bundle of $\eta$ which is not the generic situation in the $\operatorname{Spin}^{c}(4)$ case. In fact the $G$ fibres are fixed rigidly to the $\mathrm{SO}(4)$ fibres and the anticipated arbitrariness in the choice of a $G$-bundle is missing.

4.2. In general one might attempt to relate a $\operatorname{Spin}_{G}(4)$ structure to a principal $G$-bundle over $E(\xi)$ whose restriction to each $\mathrm{SO}(4)$ fibre reproduced the fibering associated with (4.2) (cf. 3.5)

$$
G \stackrel{k}{\longrightarrow} \operatorname{Spin}_{G}(4) \stackrel{\rho}{\longrightarrow} \mathrm{SO}(4), \quad k(g)=[1, g]
$$

When $G$ is $\mathbb{Z}_{2}$ or $U(1)$ this is feasible since $\mathbb{Z}_{2}$ and $U(1)$ bundles over any manifold $X$ are simply classified by, respectively, $H^{1}\left(X ; \mathbb{Z}_{2}\right)$ and $H^{2}(X ; \mathbb{Z})$. However, for general $G$ the classification problem is very complicated except when $X$ is fourdimensional or less $[3,19]$ (which $\mathrm{SO}(4)$ and $E(\xi)$ are not) or for very special choices (such as $X=\$^{n}$ ).

We can, however, develop the Puppe sequence methods in [3] to give some information on the different $\operatorname{Spin}_{G}(4)$ bundles that can cover $\xi$. In order to clarify the exposition we will present it in a sequence of steps.

1) The fibration in (4.5) leads to the derived fibration of universal classifying spaces $[5]$ :

$$
B G \stackrel{B k}{\longrightarrow} B \operatorname{Spin}_{G}(4) \stackrel{B \rho}{\longrightarrow} B \mathrm{SO}(4)
$$

An SO(4) bundle $\xi$, defined by a map $\alpha$ from $M$ into $\mathrm{BSO}(4)$, can be covered by a $\operatorname{Spin}_{G}(4)$ bundle if and only if $\alpha$ lifts to $B \operatorname{Spin}_{G}(4)$. In this case a $\operatorname{Spin}_{G}(4)$ structure is obtained with the bundle map $f$ (cf. 2.1) being obtained from the universal one associated with $B \rho[3]$.

The first four homotopy groups of $B G$ are

$$
\begin{aligned}
& \pi_{1}(B G)=0 \\
& \pi_{2}(B G)=\pi_{1}(G)=: \pi_{1} \\
& \pi_{3}(B G)=\pi_{2}(G)=0 \\
& \pi_{4}(B G)=\pi_{3}(G)=: \pi_{3}
\end{aligned}
$$

and these are sufficient to construct a Postnikov factorization $[14,15,17]$ of $(4.6)$ appropriate to a four-manifold $M$. This consists of a series of approximations to (4.6) using Eilenberg Maclane spaces to approximate $B G$. Only two levels are 
required and the construction is summarised in the diagram

$$
\begin{aligned}
K\left(\pi_{3}, 4\right) \stackrel{i_{2}}{\longrightarrow}\left(B \operatorname{Spin}_{G}(4)\right)_{4} \\
\mid{ }^{\mid p 2} \\
K\left(\pi_{1}, 2\right) \stackrel{i_{1}}{\longrightarrow}\left(B \operatorname{Spin}_{G}(4)\right)_{2} \stackrel{\theta_{2}}{\longrightarrow} K\left(\pi_{3}, 5\right) \\
\left.\right|^{p 1} \\
B \text { SO }(4) \stackrel{\theta_{1}}{\longrightarrow} K\left(\pi_{1}, 3\right)
\end{aligned}
$$

where the notation is as follows: $(X)_{n}$ is a space that is $(n+1)$ equivalent with $X$; i.e. there is a function $H: X \rightarrow(X)_{n}$ which maps the first $n$ homotopy groups isomorphically and the $(n+1)$ th group epimorphically. The maps $p_{1}$ and $p_{2}$ are the projections in the fibrations

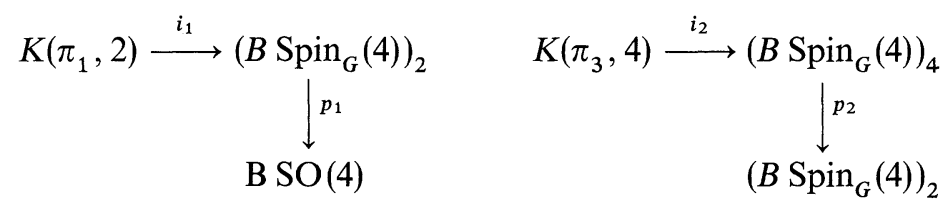

which are induced by the maps $\theta_{1}$ and $\theta_{2}$ respectively. In this context the spaces $K\left(\pi_{1}, 3\right)$ and $K\left(\pi_{3}, 5\right)$ are the base spaces of 'universal' fibrations with fibres homotopic to $K\left(\pi_{1}, 2\right)$ and $K\left(\pi_{3}, 4\right)$ respectively.

2) Mapping $M$ into the fibrations in (4.8) leads to the interlocking exact sequences

$$
\begin{aligned}
& \ldots \longrightarrow {\left[M, K\left(\pi_{3}, 4\right)\right] \stackrel{i_{2_{*}}}{\longrightarrow}\left[M,\left(B \operatorname{Spin}_{G}(4)\right)_{4}\right] \stackrel{p_{2_{*}}}{\longrightarrow} } \\
&\left.\ldots M,\left(B \operatorname{Spin}_{G}(4)\right)_{2}\right] \stackrel{\theta_{2_{*}}}{\longrightarrow}\left[M, K\left(\pi_{3}, 5\right)\right] \\
& \longrightarrow\left[M, K\left(\pi_{1}, 2\right)\right] \stackrel{i_{1_{*}}}{\longrightarrow}\left[M,\left(B \operatorname{Spin}_{G}(4)\right)_{2}\right] \stackrel{p_{1_{*}}}{\longrightarrow} \\
& {[M, B \mathrm{SO}(4)] \stackrel{\theta_{1_{*}}}{\longrightarrow}\left[M, K\left(\pi_{1}, 3\right)\right] }
\end{aligned}
$$

However i) $[M, K(\pi, n)]=H^{n}(M ; \pi)$

$$
\begin{array}{ll}
\text { ii) } H^{5}\left(M ; \pi_{3}\right)=0 & \text { if } M \text { is four dimensional } \\
\text { iii) }\left[M,(X)_{4}\right]=[M, X] & ", ", ",
\end{array}
$$

and (4.10) and (4.11) become

$$
\begin{aligned}
& \longrightarrow H^{4}\left(M ; \pi_{3}\right) \stackrel{i_{2_{*}}}{\longrightarrow} \mathscr{B}_{\operatorname{Spin}_{G}(4)}(M) \stackrel{p_{2_{*}}}{\longrightarrow}\left[M,\left(B \operatorname{Spin}_{G}(4)\right)_{2}\right] \stackrel{\theta_{2_{*}}}{\longrightarrow} 0 \\
& \longrightarrow H^{2}\left(M ; \pi_{1}\right) \stackrel{i^{i_{*}}}{\longrightarrow}\left[M,\left(B \operatorname{Spin}_{G}(4)\right)_{2}\right] \stackrel{p_{1_{*}}}{\longrightarrow} \mathscr{B}_{\mathrm{SO}(4)}(M) \stackrel{\theta^{i_{*}}}{\longrightarrow} H^{3}\left(M ; \pi_{1}\right)
\end{aligned}
$$

3) The exactness of (4.12) shows that $p_{2_{*}}$ is a surjection from $\mathscr{B}_{\operatorname{Spin}_{G}(4)}(M)$ onto $\left[M,\left(B \operatorname{Spin}_{G}(4)\right)_{2}\right]$. Thus a necessary and sufficient condition for an $\mathrm{SO}(4)$ bundle to be covered by a $\operatorname{Spin}_{G}(4)$ bundle is that $\xi$ belong to the image of $p_{1 *}$ 
(and then $p_{1_{*}}\left(p_{2_{*}}(\eta)\right)=\xi$ ) which is true if and only if $\xi$ is in the kernel of $\theta_{1_{*}}$ :

$$
\theta_{1_{*}}(\xi)=0
$$

We see that $\theta_{1 *}(\xi)$ measures the obstruction to the existence of $\operatorname{Spin}_{G}(4)$ structures. This is evidently a generalization of the $\operatorname{Spin}^{c}(4)$ case in Sect. 2 in which $\theta_{1 *}(\xi)=$ $W_{3}(\xi)$ and it is clear that for an arbitrary group $G$ and an arbitrary manifold $M$, condition (4.14) may not be satisfied. On reflection the obvious solution is to choose $G$ such that $\pi_{1}(G)=0$. The Postnikov factorization (4.8) collapses to

$$
K\left(\pi_{3}, 4\right) \stackrel{i}{\longrightarrow}\left(B \operatorname{Spin}_{G}(4)\right)_{4}
$$

leading to the single, extended, Puppe sequence (cf. 3.14)

$$
\begin{aligned}
& \ldots \longrightarrow\left.\longrightarrow, \operatorname{Spin}_{G}(4)\right] \stackrel{\Omega_{p_{*}}}{\underset{p_{*}}{\longrightarrow}}[M, \mathrm{SO}(4)] \stackrel{\Omega \theta_{*}}{\longrightarrow} H^{4}\left(M ; \pi_{3}\right) \stackrel{i_{*}}{\longrightarrow} \mathscr{B}_{\operatorname{Spin}_{G}(4)}(M) \stackrel{\theta_{*}}{\longrightarrow} 0 \\
& \stackrel{\text { S }(4)}{\longrightarrow}
\end{aligned}
$$

By exactness $p_{*}$ is surjective and hence $\operatorname{Spin}_{G}(4)$ structures always exist. From this point of view the original choice $G=U(1)$, with $\pi_{1}(G) \neq 0$, is evidently not the most appropriate.

4) The similarity between (2.12), (3.14), and (4.16) makes it tempting to conjecture that inequivalent $\operatorname{Spin}_{G}(4)$ structures are classified by the elements of $H^{4}\left(M ; \pi_{3}\right)$. However (4.16) can only immediately be employed to yield information on the number of $\operatorname{Spin}_{G}(4)$ bundles covering $\xi_{T}$ - the trivial bundle. This is (cf. (3.20))

$$
D_{\xi_{T}}=\operatorname{ker} p_{*}=\operatorname{im} i_{*}
$$

whereas the set of equivalence classes of $\mathrm{SO}(4)$ gauge functions that cannot be lifted to $\operatorname{Spin}_{G}(4)$ is (cf. (2.13)), (3.24)).

$$
\begin{aligned}
T_{\xi} & =[M, \operatorname{SO}(4)] / \operatorname{im} \Omega p_{*}=[M, S O(4)] / \operatorname{ker} \Omega \theta_{*}=\operatorname{im} \Omega \theta_{*} \\
& =\operatorname{ker} i_{*}
\end{aligned}
$$

Note that for any compact simple non abelian Lie group $\pi_{3}(G)=\mathbb{Z}$ and that on a compact oriented four manifold $H^{4}(M ; \mathbb{Z})=\mathbb{Z}$ whereas $H^{4}(M ; \mathbb{Z})=\{0\}$ if $M$ is noncompact [6]. Note also that if $\pi_{1}(G)=0$ then $(B G)_{4}=K\left(\pi_{3}, 4\right)$ and hence

$$
\mathscr{B}_{G}(M)=H^{4}\left(M ; \pi_{3}\right)
$$

and $i_{*}$ in (4.16) is essentially $B k_{*}$ (cf. (4.6)). This straightforward classification of principal bundle of simply connected groups is one of the major simplifying topological properties resulting from the dimensionality of spacetime being only four.

5) There is a natural map $\chi$ (cf. 3.8)

$$
\begin{aligned}
\chi: \operatorname{Spin}_{G}(4) & \longrightarrow G / \mathbb{Z}_{2} \\
{[x, g] } & \longmapsto[g]
\end{aligned}
$$


where $[g]$ is the image of $g$ under the canonical map $\lambda$ of $G$ onto $G / \mathbb{Z}_{2}$, and the composite of $k$ with $\chi$ is simply $\lambda$ :

$$
\begin{aligned}
G \stackrel{k}{\longrightarrow} \operatorname{Spin}_{G}(4) \stackrel{\chi}{\longrightarrow} G / \mathbb{Z}_{2} \\
g \longmapsto[1, g] \longmapsto[g]
\end{aligned}
$$

This is associated with the fibrations

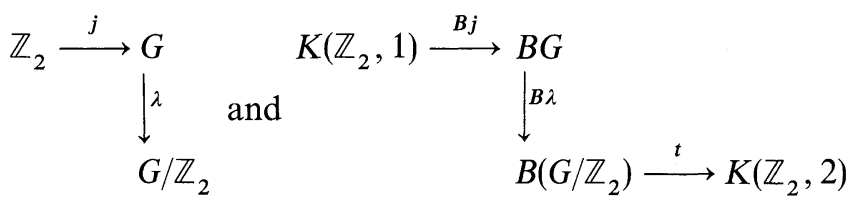

which induces the exact sequence (using (4.19))

$$
\ldots \longrightarrow H^{1}\left(M ; \mathbb{Z}_{2}\right) \stackrel{B j_{*}}{\longrightarrow} H^{4}\left(M ; \pi_{3}\right) \stackrel{B \lambda_{*}}{\longrightarrow} \mathscr{B}_{G / \mathbb{Z}_{2}}(M) \stackrel{t_{*}}{\longrightarrow} H^{2}\left(M ; \mathbb{Z}_{2}\right)
$$

Now $B j_{*}$ is derived from a map from $K\left(\mathbb{Z}_{2}, 1\right) \rightarrow K\left(\pi_{3}, 4\right)=(B G)_{4}$ whose homotopy classes are classified by elements of $H^{4}\left(K\left(\mathbb{Z}_{2}, 1\right) ; \mathbb{Z}\right)=\mathbb{Z}_{2}[14]$ when $G$ is simple (the extension to semi-simple $G$ is obvious). The nontrivial element in $\mathbb{Z}_{2}$ corresponds to the map

$$
B j_{*}(c)=\beta(c) \cup \beta(c) \quad \forall c \in H^{1}\left(M ; \mathbb{Z}_{2}\right)
$$

where $\beta$ is the Bockstein map (3.21) from $H^{1}\left(M ; \mathbb{Z}_{2}\right)$ into $H^{2}(M ; \mathbb{Z})$. However $2 \beta=0$ and hence $2 B j_{*}=0$ which, when $M$ is compact and orientable, implies $B j_{*}=0$ since $H^{4}(M ; \mathbb{Z})=\mathbb{Z}$ has no 2-torsion. Thus in (4.23) ker $B \lambda_{*}=0$. Strictly speaking (as mentioned previously in connection with step 4) this only implies that $B \lambda_{*}$ is one to one onto the trivial bundle. In general two $G$ bundles represented by elements $p_{1}$ and $p_{2}$ in $H^{4}(M ; \mathbb{Z})$, will map onto the same $G / \mathbb{Z}_{2}$ bundle if and only if they are related by a certain action of $H^{1}\left(M ; \mathbb{Z}_{2}\right)$ on $H^{4}(M ; \mathbb{Z})$ associated with the fibration in (4.22). However the argument which leads to (4.24) may be readily extended and shows that in all cases the map $B \lambda_{*}$ is injective.

Now $\lambda=\chi \circ k$ and hence $B \lambda=B \chi \circ B k$ which implies

$$
B \lambda_{*}=B \chi_{*} \circ B k_{*}
$$

Thus $B \lambda_{*}$ injective implies that $B k_{*}$ is injective in (4.16). Thus

$$
\begin{aligned}
D_{\xi_{T}} & =H^{4}(M ; \mathbb{Z}) \\
T_{\xi} & =\{0\}
\end{aligned}
$$

and it seems very likely that (4.26) remains true for all SO(4) bundles.

In summary we see that $\operatorname{Spin}_{G}(4)$ structures always exist provided that $G$ is any simply connected, simple, Lie group and that the different $\operatorname{Spin}_{G}(4)$ bundles which can cover the given $\mathrm{SO}(4)$ bundle are labelled by elements of $H^{4}(M ; \mathbb{Z})=\mathbb{Z}$. Furthermore as shown by (4.27), $\mathrm{SO}(4)$ valued 'gauge' functions may always be lifted to $\operatorname{Spin}_{G}(4)$.

4.3. It may not be transparent from the arguments above how a suitable $\operatorname{Spin}_{G}(4)$ bundle is actually constructed. There is an alternative way of looking at $\operatorname{Spin}_{G}(4)$ 
structures which clarifies this point and throws more light on the situation:

1) The projections (4.2) and (4.20) may be combined to give the fibre bundle

$$
\mathbb{Z}_{2} \longrightarrow \operatorname{Spin}_{G}(4) \stackrel{p}{\longrightarrow} \mathrm{SO}(4) \times\left(G / \mathbb{Z}_{2}\right)
$$

with $p[x, g]=(\Lambda(x),[g])$. This suggests that it might be possible to build a $\operatorname{Spin}_{G}(4)$ structure by forming a $\mathbb{Z}_{2}$ covering over the direct sum of the $\mathrm{SO}(4)$ bundle $\xi$ and some suitably chosen $G / \mathbb{Z}_{2}$ bundle $\zeta$. Two such structures $(\eta, \varphi)$ and $\left(\eta^{\prime} . \varphi^{\prime}\right)$ are defined to be $\mathbb{Z}_{2}$ equivalent if there exists a $\operatorname{Spin}_{G}(4)$ bundle map $g: \eta \rightarrow \eta^{\prime}$ making the diagram

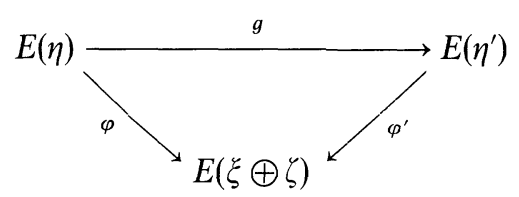

commute. Now $H^{1}\left(\mathrm{SO}(4) \times G / \mathbb{Z}_{2} ; \mathbb{Z}_{2}\right)=\mathbb{Z}_{2} \oplus \mathbb{Z}_{2}$ and there are correspondingly four double coverings of $\mathrm{SO}(4) \times G / \mathbb{Z}_{2}: \mathrm{SO}(4) \times G / \mathbb{Z}_{2} \times \mathbb{Z}_{2}, \operatorname{Spin}(4) \times G / \mathbb{Z}_{2}$, $\mathrm{SO}(4) \times G$, and $\operatorname{Spin}_{G}(4)$.

By analogy with the Spin(4) case we must clearly look for a double covering of $E(\xi \oplus \zeta)$ which, when restricted to each $\mathrm{SO}(4) \times G / \mathbb{Z}_{2}$ fibre, reproduces the $\operatorname{Spin}_{G}(4)$ covering. The relevant Serre cohomology sequence is (cf. 2.4))

$$
\begin{aligned}
0 \longrightarrow H^{1}\left(M ; \mathbb{Z}_{2}\right) \longrightarrow H^{2}\left(E(\xi \oplus \zeta) ; \mathbb{Z}_{2}\right) \longrightarrow H^{2}\left(\mathrm{SO}(4) \times G / \mathbb{Z}_{2} ; \mathbb{Z}_{2}\right) \\
\stackrel{{ }^{\imath}}{\longrightarrow} H^{2}\left(M ; \mathbb{Z}_{2}\right)
\end{aligned}
$$

and we require

$$
\tau(l)=0
$$

where $t$ is the element in $H^{1}\left(\mathrm{SO}(4) \times G / \mathbb{Z}_{2} ; \mathbb{Z}_{2}\right)$ corresponding to the $\operatorname{Spin}_{G}(4)$ covering.

2) Alternatively (4.28) gives the fibration

$$
\begin{aligned}
K\left(\mathbb{Z}_{2}, 1\right) \longrightarrow & B \operatorname{Spin}_{G}(4) \\
& B\left(\operatorname{SO}^{\mid}(4) \times G / \mathbb{Z}_{2}\right) \stackrel{\theta}{\longrightarrow} K\left(\mathbb{Z}_{2}, 2\right)
\end{aligned}
$$

and the exact sequence

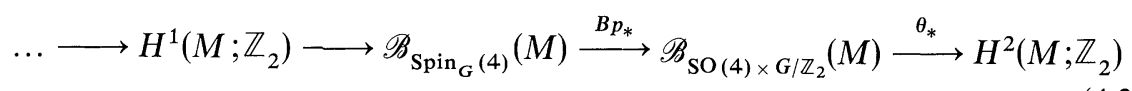

and the condition for a covering is

$$
\theta_{*}(\xi \oplus \zeta)=0
$$


3) The classification of $G / \mathbb{Z}_{2}$ bundles may be probed by the Postnikov factorization

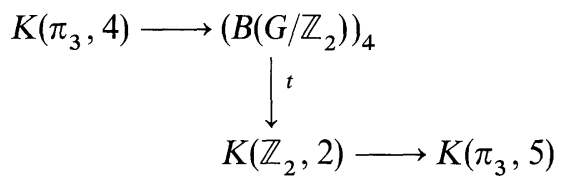

and associated exact sequence

$$
\ldots \longrightarrow H^{1}\left(M ; \mathbb{Z}_{2}\right) \longrightarrow H^{4}\left(M ; \pi_{3}\right) \longrightarrow \mathscr{B}_{G / \mathbb{Z}_{2}}(M) \stackrel{t_{*}}{\longrightarrow} H^{2}\left(M ; \mathbb{Z}_{2}\right) \longrightarrow 0
$$

which is simply an extension of (4.23). Evidently $t_{*}(\zeta)$ is a characteristic class of the bundle and indeed, if $G=\operatorname{Spin}(n)$, then $t_{*}(\zeta)=w_{2}(\zeta)$.

It is easily shown that

$$
\tau(i)=\theta_{*}(\xi \oplus \zeta)=w_{2}(\xi)+t_{*}(\zeta)
$$

and so in order to lift $\xi \oplus \zeta$ to a $\operatorname{Spin}_{G}(4)$ bundle we require

$$
t_{*}(\zeta)=\omega_{2}(\xi)
$$

However (4.35) shows that $t_{*}$ is surjective and hence (4.37) can always be satisfied. Note that again this is a special property of 4-manifolds $M$ since $H^{5}\left(M ; \pi_{3}\right)=0$.

Thus we see that a constructive way of building a $\operatorname{Spin}_{G}(4)$ structure is to choose a $G / \mathbb{Z}_{2}$ bundle whose characteristic $H^{2}\left(M ; \mathbb{Z}_{2}\right)$ class kills the second Stiefel Whitney class of $\xi$ and then form an appropriate $\mathbb{Z}_{2}$ covering of the product bundle. The $G / \mathbb{Z}_{2}$ bundle may be chosen arbitrarily subject only to (4.37). In particular the $H^{4}\left(M ; \pi_{3}\right)$ classifying elements are freely specifiable within the restrictions imposed on any $G / \mathbb{Z}_{2}$ bundle (for example the first Pontryagin class $p$ of a $\mathrm{SO}(n)$ bundle must satisfy $\left.p(\zeta) \bmod 2=w_{2}(\zeta) \cup w_{2}(\zeta)\right)$.

Thus there will be $H^{4}\left(M ; \pi_{3}\right)$ 'winding numbers' associated with the gauged $G / \mathbb{Z}_{2}$ group and these can be expected to manifest themselves in the quantum field theory in the usual instanton like fashion in which a real characteristic class is represented by a connection in the bundle $\zeta$. This may be combined with a linear connection on $\xi$ to give a connection on the $\operatorname{SO}(4) \times G / \mathbb{Z}_{2}$ bundle which in turn will be lifted up onto the covering $\operatorname{Spin}_{G}(4)$ bundle and used in the construction of covariant derivatives of the generalized spinors.

\section{Conclusion}

We have analysed the existence and uniqueness of generalized spin structures using two essentially different techniques. The first of these involved lifting the structure group directly from $\mathrm{SO}(4)$ to $\operatorname{Spin}_{G}(4)$ through the projection map $\rho$. The second method formed the Whitney sum $\xi \oplus \zeta$ where $\zeta$ was a suitable $G / \mathbb{Z}_{2}$ bundle and then attempted to double cover the structure group to $\operatorname{Spin}_{G}(4)$. Associated with these two methods were two different notions of equivalence of $\operatorname{Spin}_{G}(4)$ structures. This difference leads to slight differences in the resulting 
classification schemes. Specifically, given a generalized spin structure of either sort it determines a structure of the other type, but the correspondence is generally not bijective. To see this consider the commutative diagram of principal bundle maps

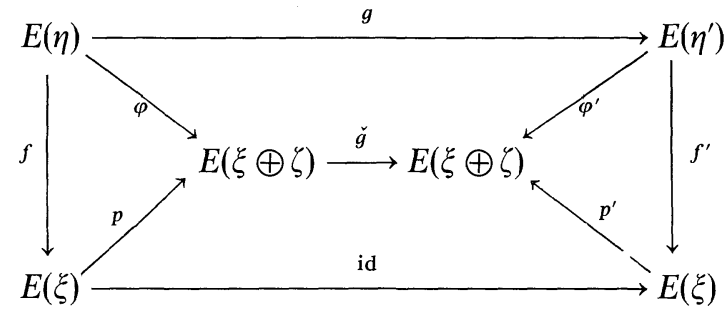

Thus if $(\eta, \varphi)$ and $\left(\eta^{\prime}, \varphi^{\prime}\right)$ are $\mathbb{Z}_{2}$-equivalent then taking $\breve{g}=$ id shows that $(\eta, f)$ and $\left(\eta^{\prime}, f^{\prime}\right)$ are equivalent. However, if $(\eta, f)$ and $\left(\eta^{\prime}, f^{\prime}\right)$ are equivalent then $\breve{g}$ need not necessarily be the identity. In other words the difference between the two classification schemes is quantified by those equivalence classes of automorphisms of $\xi \oplus \zeta$ which do not lift to $\eta$. This is clearly shown in the $U(1)$ case where the first approach gave $\left|H^{2}(M ; \mathbb{Z})\right|=|\operatorname{im} 2| \cdot|\operatorname{im} \beta|$ different structures whilst the second gave $|\operatorname{im} 2| \cdot\left|H^{1}\left(M ; \mathbb{Z}_{2}\right)\right|$ structures. These numbers are not generally the same: they differ by a factor $|\operatorname{ker} \beta|$. A similar result presumably holds for general $G$. The physical implications of these definitions of equivalence will be the subject of a future paper.

In both approaches the basic idea is to kill the non vanishing second Stiefel Whitney class of the tangent bundle with the characteristic $H^{2}\left(M ; \mathbb{Z}_{2}\right)$ class of a suitably chosen $G / \mathbb{Z}_{2}$ bundle $\zeta$. It would be interesting to take a specific space such as $\mathbb{C P}{ }_{2}$ and work out some of the $G / \mathbb{Z}_{2}$ instanton solutions which are doubtless associated with the construction. Quantum field theoretic quantities such as the axial current anomaly should be relatable to the characteristic classes of $\xi$ and $\zeta$. This will present some features that, compared to the usual $\mathbb{\$}^{4}$ case, are rather novel and which arise because of the presence of the $H^{2}\left(M ; \mathbb{Z}_{2}\right)$ classes.

It should be noted that our schemes will, like the original method of Hawking and Pope [8], lead to relations between the spin and internal symmetry numbers. These arise because representations of $\operatorname{Spin}_{G}(4)$ are constructed from $\operatorname{Spin}(4) \times G$ representations that are trivial on the $\mathbb{Z}_{2}$ subgroup.

The restrictions on $G$ are not without interest. They permit the use of $\mathrm{SU}(2 n)(n=1,2,3 \ldots)$ but not $\mathrm{SU}(2 n+1)$. Another obvious choice is $\operatorname{Spin}(n)$ $(n \geqq 3)$ with $G / \mathbb{Z}_{2}=\mathrm{SO}(n)$. Historically the use of an orthogonal internal symmetry group is rather unusual. However, the advent of extended supergravity theories has changed this as they are based predominantly on such groups. Spin $(n)$ based generalised spin structures could therefore be of considerable use if an attempt is ever made to construct extended supergravity models on spaces with non vanishing second Stiefel Whitney class. 


\section{References}

1. Atiyah, M. F., Bott, R., Patodi, V. K. : Invent. Math. 19, 279-330 (1973)

2. Avis, S. J., Isham, C. J. : Nucl. Phys. B156, 441 (1979)

3. Avis, S. J., Isham, C. J. : Proceedings of the 1978 Cargèse Summer School on recent developments in general relativity (eds. S. Deser, M. Levy). London, New York: Plenum 1979

4. Back, A., Freund, P. G. O., Forger, M. : Phys. Lett. 77B, 181-184 (1978)

5. Borel, A. : Topics in the homology theory of fibre bundles. In: Lecture notes in mathematics Vol. 36. Berlin, Heidelberg, New York: Springer 1967

6. Dold, A. : Lectures on algebraic topology. Berlin, Heidelberg, New York : Springer 1972

7. Forger, M., Hess, H. : Commun Math. Phys. 64, 269-278 (1979)

8. Hawking, S. W., Pope, C. N. : Phys. Lett. 73B, 42-44 (1978)

9. Hirzebruch, F.: Topological methods in algebraic topology. Berlin, Heidelberg, New York: Springer 1966

10. Hitchin, N. J. : Adv. Math. 14, 1-55 (1974)

11. Husemoller, D. : Fibre bundles. Berlin, Heidelberg, New York: Springer 1966

12. Isham, C. J. : Proc. R. Soc. 364A, 591-599 (1978)

13. Milnor, J. : L'Ens. Math. 9, 198-203 (1963)

14. Mosher, R. E., Tangora, M. C. : Cohomology operations and applications in homotopy theory. New York, London: Harper Row 1968

15. Spanier, E. H. : Algebraic topology. New York: McGraw-Hill 1966

16. Steenrod, N. : The topology of fibre bundles. Princeton: Princeton University Press 1951

17. Thomas, E. : Seminar on fibre spaces. In: Lecture notes in mathematics, Vol. 13. Berlin, Heidelberg, New York: Springer 1976

18. Whiston, G. S. : Gen. Relativ. Grav. 6, 463-475 (1975)

19. Dold, A., Whitney, H.: Ann. Math. 69, 3 (1959)

Communicated by R. Geroch

Received June 19, 1975 